Neurobiol Aging. 2013 November ; 34(11): 2510-2516. doi:10.1016/j.neurobiolaging.2013.05.017.

\title{
Striatal shape in Parkinson's disease
}

\author{
Nicholas W. Sterling, B.S. ${ }^{*}, \mathrm{ab}$, Guangwei Du, M.D., Ph.D. ${ }^{\star}, \mathrm{a}$, Mechelle M. Lewis, Ph.D. ${ }^{\mathrm{a}, \mathrm{c}}$, \\ Christopher Dimaio, B.S. ${ }^{a}$, Lan Kong, Ph.D. ${ }^{b}$, Paul J. Eslinger, Ph.D. ${ }^{a, b, d}$, Martin Styner, \\ Ph.D.g, and Xuemei Huang, M.D., Ph.D. ${ }^{a, c, d, e, f}$ \\ aDepartment of Neurology, Pennsylvania State University-Milton S. Hershey Medical Center, \\ Hershey PA 17033, USA \\ bDepartment of Public Health Sciences, Pennsylvania State University-Milton S. Hershey Medical \\ Center, Hershey PA 17033, USA \\ 'Department of Pharmacology, Pennsylvania State University-Milton S. Hershey Medical Center, \\ Hershey PA 17033, USA \\ dDepartment of Radiology, Pennsylvania State University-Milton S. Hershey Medical Center, \\ Hershey PA 17033, USA \\ eDepartment of Neurosurgery, Pennsylvania State University-Milton S. Hershey Medical Center, \\ Hershey PA 17033, USA \\ fDepartment of Kinesiology, Pennsylvania State University-Milton S. Hershey Medical Center, \\ Hershey PA 17033, USA
}

9Department of Computer Science, University of North Carolina, Chapel Hill, NC 27599, USA

\section{Abstract}

Parkinson's disease (PD) is marked pathologically by nigrostriatal dopaminergic terminal loss. Histopathological and in vivo labeling studies demonstrate that this loss occurs most extensively in the caudal putamen and caudate head. Previous structural studies have suggested reduced striatal volume and atrophy of the caudate head in PD subjects. The spatial distribution of atrophy in the putamen, however, has not been characterized. We aimed to delineate the specific locations of atrophy in both of these striatal structures. T1- and T2-weighted brain MR (3T) images were obtained from $40 \mathrm{PD}$ and 40 control subjects having no dementia and similar age and gender distributions. Shape analysis was performed using doubly segmented regions of interest. Compared to controls, PD subjects had lower putamen $(\mathrm{p}=0.0003)$ and caudate $(\mathrm{p}=0.0003)$ volumes. Surface contraction magnitudes were greatest on the caudal putamen ( $\mathrm{p} \unlhd$ ().005) and head and dorsal body of the caudate ( $\mathrm{p} \unlhd$.005). This spatial distribution of striatal atrophy is consistent with the known pattern of dopamine depletion in PD and may reflect global consequences of known cellular remodeling phenomena.

\footnotetext{
(C) 2013 Elsevier Inc. All rights reserved.

Corresponding Author: Xuemei Huang M.D., Ph.D., Associate Professor of Neurology, Penn State Hershey Medical Center, 500 University Dr., H-037, Hershey, PA 17033-0850, USA, Voice: 717-531-1803 Fax: 717-531-0226, xhuang @ psu.edu. *These authors contributed equally to this work.

Publisher's Disclaimer: This is a PDF file of an unedited manuscript that has been accepted for publication. As a service to our customers we are providing this early version of the manuscript. The manuscript will undergo copyediting, typesetting, and review of the resulting proof before it is published in its final citable form. Please note that during the production process errors may be discovered which could affect the content, and all legal disclaimers that apply to the journal pertain.

Disclosure Statement

The authors report no conflicts of interest.
} 


\section{Keywords}

striatum; putamen; caudate; Parkinson's disease; shape; volume; structure; morphology; magnetic resonance imaging (MRI)

\section{Introduction}

Parkinson's disease (PD) is the second most common age-related neurodegenerative disorder and is characterized clinically by tremor at rest, bradykinesia, and rigidity (de Lau and Breteler, 2006). Pathologically, PD is marked by loss of dopaminergic neurons in the substantia nigra pars compacta of the midbrain (Jellinger, 2012; Braak et al., 2003). Dopamine depletion of the striatum (putamen and caudate), resulting from the loss of nigrostriatal terminals, reaches $60-80 \%$ before the onset of classic motor symptoms, most severely affecting the caudal portion of the putamen and head of the caudate (Kish et al., 1988; Rodriguez-Oroz et al., 2009; Otsuka et al., 1996; Lang and Obeso, 2004).

Previous studies have suggested that the striatum undergoes substantial cellular remodeling throughout the course of PD progression. Dopamine-depleted animal and postmortem studies of PD subjects, for example, have demonstrated reduced dendritic length and spine density of the medium spiny neurons within the striatum (Ingham et al., 1993; Stephens et al., 2005; McNeill et al., 1988). In humans, spine density is reduced particularly in the caudal part of the putamen, where dopaminergic deficits are known to be most severe (ZajaMilatovic et al., 2005). Moreover, dopamine is known to play an important role in maintaining striatal spine integrity (Villalba et al., 2009).

Several structural studies have suggested that the putamen and caudate undergo atrophy in PD (for review, see Pitcher et al., 2012). One previous study by Apostolova et al. (2010) demonstrated trend-level shape differences in the caudate between non-demented PD and control subjects. The spatial distribution of atrophic areas of the putamen, however, remains uncharacterized. Information regarding the pattern of striatal atrophy is important because it may yield insight into the structural consequences of striatal cellular remodeling and also may provide region-specific biomarkers that reflect PD disease progression. In this study, we aimed to characterize these atrophic sites using a state-of-the-art protocol for segmentation followed by shape analysis using spherical harmonic point distribution models (SPHARM-PDM) (Styner et al., 2005). The present study included only non-demented subjects in order to avoid potentially confounding influences of dementia-related neuropathology. We hypothesized that striatal shape differences would be greatest in areas that are known to be affected most severely by dopamine depletion and consequent striatal spine pathology in PD, namely, the caudal portion of the putamen.

\section{Methods}

\subsection{Subjects}

Forty PD subjects ( 20 female, 20 male) and 40 healthy control subjects ( 20 female, 20 male) having similar age distributions (61.3 \pm 7.8 years for PD subjects, $59.4 \pm 7.8$ years for control subjects) were recruited from a tertiary movement disorders clinic. PD diagnosis was confirmed by a movement disorders specialist using published criteria (Hughes et al., 1992). All subjects were confirmed for absence of other major and acute neurological and psychological disorders, hypothyroidism, vitamin $\mathrm{B}_{12}$ and folate deficiency, and kidney and liver disease. Unified Parkinson's Disease Rating Scale section III motor scores (UPDRSIII) were obtained for each subject, with PD subjects assessed after withholding PD medications overnight ( $>12$ hours) as a practically-defined off-drug state (Langston et al., 
1992). Contralateral and ipsilateral sides were defined, respectively, as opposite to the body sides having the greater and lesser UPDRS-III motor scores. Duration of illness (DOI) was determined based on date of clinical diagnosis. Levodopa equivalent daily dose (LEDD) was calculated using published criteria (Tomlinson et al., 2010). Written informed consent was obtained for each subject, in accordance with the Declaration of Helsinki. The research protocol was reviewed and approved by the Penn State Hershey Medical Center Institutional Review Board.

\subsection{Neuropsychological assessment}

All subjects completed a basic neuropsychological battery to assess orientation, memory, and symptoms of dementia. The Mini Mental State Exam (MMSE) is a brief (5-minute) 30point test that assesses orientation, memory and the ability to follow commands (Folstein et al., 1975). The MMSE has been shown to have $98 \%$ sensitivity and $77 \%$ specificity in detecting dementia among PD subjects using a cutoff score of 23 (Hobson and Meara, 1999). The Montreal Cognitive Assessment (MoCA) was developed to address the limitations of the MMSE in detecting mild cognitive impairment (MCI) (Hoops et al., 2009) and has been shown to have higher sensitivity for detecting PD-MCI than the MMSE (Marras et al., 2013). The Dementia Rating Scale-2 (DRS2) examines a broader range of cognitive functions (attention, initiation and perseveration, conceptualization, construction, and memory) and has been demonstrated to be sensitive for detecting cognitive impairments in PD (Brown et al., 1999). In this study, the cutoff for dementia was set at the $5^{\text {th }}$ percentile (i.e., 1.67 standard deviations below the mean), in line with recommended guidelines (Monsch et al., 1995; Jurica et al., 2004). Subjects were included in this study only if they had both an MMSE score $>24$ and a DRS2 score $>5^{\text {th }}$ percentile to ensure they were not demented.

\subsection{Image acquisition}

All subjects were scanned using a 3.0 Tesla MR scanner (Trio, Siemens Magnetom, Erlangen, Germany, with an 8-channel phased array head coil) and high-resolution T1- and T2-weighted images were acquired. A magnetization-prepared rapid acquisition gradient echo sequence was used to obtain T1-weighted images with $\mathrm{TR}=1540 \mathrm{~ms}, \mathrm{TE}=2.34 \mathrm{~ms}$, field of view $=256 \mathrm{~mm}$, matrix $=256 \times 256$, slice thickness $=1 \mathrm{~mm}$ (with no gap), and slice number $=176$. T2-weighted images were collected using a fast-spin-echo sequence with $\mathrm{TR}=2500, \mathrm{TE}=316$, and the same resolution configuration as that for T1-weighted images.

\subsection{Semi-automatic segmentation}

Putamen and caudate structures were obtained using a semi-automatic region of interest (ROI)-based approach. Probabilistic atlas-based automatic segmentation first was performed using AutoSeg (Neuro Image Research and Analysis Laboratories, University of North Carolina at Chapel Hill, NC, USA). This software features N4 bias field correction, expectation-maximization tissue segmentation, skull striping, and probabilistic atlas-based segmentation of subcortical structures (Lewis et al., 2009; Vachet et al., 2001; Van et al., 1999; Van Leemput et al., 1999). The resulting ROIs then were manually corrected by an investigator (NWS) blinded to subject diagnosis and symptoms using ITK-SNAP 2.2.0 (www.itksnap.org) (Yushkevich et al., 2006). In order to establish consistent boundaries of the caudate and putamen, and to exclude the nucleus accumbens, manual corrections were performed following the anatomical guidelines available in the UNC NeuroImage Analysis Lab Manual (2007). In addition to the utilization of these guidelines for manual correction of the striatal structures, the lateral ventricles also were segmented using the level-set regiongrowing tool in ITK-SNAP, in order to maximize the accuracy of the caudate-ventricle borders. To avoid potential left-right rater bias and increase the consistency of the final ROIs, this semi-automated process was repeated after a delay of four weeks using a mirrored 
version of the original image set [in which left and right sides were inverted (Maltbie et al., 2012)].

\subsection{Volume extraction \& average regions of interest}

Volumes for each ROI were extracted from mirrored and original image sets and then were averaged together, maintaining original left and right designations. Average ROIs from the original and mirrored ROI sets also were generated for subsequent shape analysis using a Gaussian averaging process. Mirrored ROIs first were reverted to their original orientations. Subsequently, a Gaussian smoothing process (standard deviation $=0.3 \mathrm{~mm}$ ) was applied to the original and reverted mirrored ROIs. Intensity values of original and mirrored ROIs were summed at each corresponding voxel and then scaled so that the maximum and minimum intensities per combined ROI were 1 and 0 , respectively. In order to yield average ROIs having solid borders and fill, the combined ROIs then were binarized using an intensity threshold of 0.8 . This process of averaging the original and reverted mirrored ROIs was performed in order to increase the accuracy of each shape and to correct for the known phenomenon of asymmetric (right-left) rater bias (Maltbie et al., 2012).

\subsection{Mesh generation, spherical parameterization, and alignment}

Shape analysis was performed on the averaged ROIs using the SPHARM-PDM (Spherical Harmonics Point Distribution Models) toolbox [Neuro Image Research and Analysis Laboratories, University of North Carolina at Chapel Hill, NC, USA; (Styner et al., 2005; Styner et al., 2003; Styner,M. et al., 2006b)]. The ROIs first were processed in order to fill any internal holes and adapted to guarantee spherical topology. Surface meshes and corresponding spherical parameterizations then were computed using area-preserving, distortion minimizing spherical mapping. The SPHARM descriptions were generated from surface meshes and their spherical parameterizations. The first order ellipsoids from the spherical harmonic coefficients were used to establish initial correspondence between the spherical parameterizations and each model was co-registered via generalized rigid Procrustes alignment. The SPHARM descriptions then were sampled into surface meshes with each of the 1,442 surface points by icosahedron subdivision. All models were scaled to normalize for intracranial cavity volume differences. Models were inspected visually by an independent investigator $(\mathrm{ZN})$ for anatomical accuracy and proper alignment.

\subsection{Statistical analyses}

Demographic and clinical parameters were compared using Student's t-tests. Group comparisons of volumes (scaled as a percent of total intracranial volume) were performed by analysis of covariance with adjustment for age and gender. Contralateral and ipsilateral scaled volumes of PD subjects having unequal left and right UPDRS-III motor scores were compared to the bilateral average of left and right volumes of all control subjects. Partial correlation coefficients between clinical measurements and scaled striatal volumes were computed after adjustment for age and gender effects. All volume analyses were performed using SAS 9.3.

Group shape differences were analyzed using the multivariate analysis of covariance (MANCOVA) tool that is part of the SPHARM-PDM toolkit (Styner,M. et al., 2006a; Paniagua et al., 2009). Specifically, shape statistics were calculated by treating the threedimensional spatial coordinates as multivariate response variables at each surface point, and by general linear modeling and permutation-based testing of the local Hotelling trace, adjusted for age and gender. Vector field maps representing the displacement between the PD and control models were generated by computing the distance and direction between group mean surfaces at each pair of corresponding points (Paniagua et al., 2009). Pearson correlation analyses of MoCA scores and shapes were performed for each surface point 
using general linear modeling of the distance from average surface (signed positively for surface expansion and negatively for contraction) and MoCA residuals of regression on age and gender. Topographical statistical significance maps for each surface coordinate were generated after correction for multiple comparisons using false-discovery rate (FDR) adjustment. Reported p-values for shape analyses were adjusted via the Benjamini-Hochberg FDR p-value adjustment procedure using an expected proportion of incorrectly rejected null hypotheses of 5\% (Yekutieli and Benjamini, 1999). Points having an unadjusted p-value less than or equal to the critical value were deemed real effects and were adjusted while others were assigned p-values of 1 .

\section{Results}

\subsection{Study subjects \& overlap of segmentations}

Detailed subject characteristics and clinical parameters are summarized in Table 1. There were no significant differences in age or cognitive assessment scores between PD and control groups. In PD subjects, the mean time since diagnosis was 4.1 years with a range of $0.1-15.8$ years. LEDD varied widely in PD subjects, ranging from $0-1,975 \mathrm{mg}$.

Analysis of overlap between the manually corrected original and reverted mirrored ROIs was performed by computing the Dice coefficient of mean overlap for each striatal structure (Tustison and Gee, 2009). The mean and standard deviations of overlap for left caudate, right caudate, left putamen, and right putamen, respectively, were $0.9053 \pm 0.0234$, $0.9062 \pm 0.0294,0.9006 \pm 0.0279$, and $0.9060 \pm 0.0225$.

\subsection{Volume comparisons between PD and control striatal structures}

To account for possible effects of disease-related asymmetry, contralateral and ipsilateral striatal structures were first compared in subjects having unequal left and right UPDRS-III scores. This analysis revealed no asymmetry in volumes. Thus, striatal volumes were averaged bilaterally for each subject and the mean volume was used in subsequent analyses.

PD subjects had significantly lower scaled striatal volumes (Table 2) than control subjects. Average putamen volumes in PD subjects were $7.1 \%$ lower $(\mathrm{p}=0.0003)$ than controls and caudate volumes were $7.0 \%$ lower ( $\mathrm{p}=0.0003$ ). Subgroup analysis of PD subjects with shorter durations of illness (lowest 50\% and lowest 25\%) compared to controls showed similar results (Table 2). Comparison of contralateral and ipsilateral volumes with bilaterally averaged control volumes revealed significant differences for the putamen and caudate, as did the unilateral comparisons of left and right volumes between PD and control subjects (Supplemental Table 1).

\subsection{Clinical correlations of striatal volumes in PD subjects}

In PD subjects, scaled putamen volume correlated positively with MoCA scores after adjustment for age and gender effects (Table 3). This relationship remained significant after correction for multiple comparisons $(\mathrm{p}=0.0072)$. The correlation between scaled caudate volume and MoCA scores revealed only a marginally significant relationship before correction for multiple comparisons. Age and gender adjusted correlations between scaled volume, MMSE, and DRS2 scores were not significant.

\subsection{Shape comparisons of PD and control striatal structures}

Contralateral and ipsilateral sides first were compared within the PD group for 36 subjects having unequal left and right UPDRS-III scores. Similar to the volume analysis above, there were no differences in shape between contralateral and ipsilateral structures. Thus, left and right putamen and caudate models were pooled to compare PD and control shapes. 
Comparison of PD and control striatal shapes after adjustments for age and gender revealed significant differences. For the putamen, the most statistically significant differences were localized to the caudal and ventrolateral areas ( $\mathrm{p} \unlhd 0.005$; Figure 1). Inspection of the displacement vector field map (Paniagua et al., 2009) revealed that the magnitude of surface contraction was greatest in the caudal portion of the putamen (Figure 1, Supplemental Figure 1). For the caudate, the most significant shape differences were present in the head and dorsal body ( $\mathrm{p} \unlhd .005$; Figure 2). The greatest magnitudes of caudate surface contraction were located at the most rostral extent of the caudate head (Figure 2, Supplemental Figure 2). Subgroup analysis comparing striatal shapes in all controls and PD subjects having shorter durations of illness (lowest 50\% and 25\%) showed similar results.

Although no shape differences were found between contralateral and ipsilateral structures in PD subjects, comparison of these shapes with pooled left and right control structures revealed significant differences in the caudal regions of the contralateral and ipsilateral putamen. The FDR-adjusted p-value maps revealed that, compared to the ipsilateral putamen, a greater proportion of points on the caudal contralateral putamen were separated significantly between groups (Supplemental Figure 3).

\subsection{Clinical correlations of striatal shape in PD subjects}

After the determination that MoCA was the only clinical measurement that was significantly correlated with striatal volume, we focused our shape correlation analysis on this measurement. Indeed, we found that MoCA scores, adjusted for age and gender, correlated significantly with the signed distance from average surface on the dorsolateral and rostral ventrolateral surfaces of the putamen ( $\mathrm{p} \unlhd \mathbf{\Delta} .005$; Figure 3 ) and the body and dorsal surfaces of the caudate ( $\mathrm{p} \unlhd .005$; Figure 4). We also explored potential striatal shape correlations with other clinical measurements. No significant correlations between striatal shape and other clinical measurements were observed after FDR-adjustment.

\section{Discussion}

The current data are consistent with previous reports of striatal atrophy in PD and indicate that striatal shape differences between PD and control subjects are most robust in the caudal part of the putamen and caudate head. These shape differences were present even in recently diagnosed PD patients. To our knowledge, this is the first study demonstrating PD-related shape differences in the putamen. Consistent with our hypothesis, these region-specific shape differences are reflective of the known differential pattern of striatal dopamine depletion and associated spine loss in PD (Kish et al., 1988; Rodriguez-Oroz et al., 2009; Otsuka et al., 1996; Zaja-Milatovic et al., 2005). It is possible, therefore, that disruption of dopaminergic innervation may play a role in the relatively early phenomenon of PD-related striatal atrophy.

\subsection{Technical innovations}

Apostolova et al. (2010) studied shape differences in PD subjects across various stages of cognitive decline and reported some differences in caudate shape between PD subjects (12 with normal cognition, 8 with mild cognitive impairment, and 15 with dementia) and 20 healthy controls. The current study differed substantially from that of Apostolova et al. (2010) in terms of sample characteristics and methodologies. First, in order to evaluate processes occurring relatively early in PD progression and exclude any confounds related to dementia, we included only non-demented PD subjects. The average duration of disease among the present PD subjects (4.1 years) also was much shorter [> 10 years in the report of Apostolova et al. (2010)]. Second, Apostolova et al. (2010) used a machine learning approach for segmentation, whereas we utilized a semi-automated ROI-based segmentation 
protocol that supplements the consistency of an atlas-based approach with the anatomic validity of manually defined ROIs. Third, we controlled for the known phenomenon of leftright rater segmentation bias by averaging original and left-right mirrored ROI sets (Maltbie et al., 2012). Lastly, the present study utilized SPHARM-PDM (Styner et al., 2005) in order to quantify shape differences, whereas the study by Apostolova et al. (2010) used radial distance mapping (Thompson et al., 2004). Although radial distance has been used previously to quantify putamen shape (Becker et al., 2011), the SPHARM-PDM shape analysis technique provides direct modeling of surface information and local alignment of shape.

\subsection{Striatal shape characteristics and known cellular mechanisms}

The current study is consistent with the findings of previous volume studies that suggest striatal atrophy in PD (see review by Pitcher et al., 2012). Although the exact mechanisms of volume and shape differences remain unknown, it is possible that striatal atrophy is linked, at least in part, to cellular changes within the striatum that are triggered by inadequate or fluctuating levels of dopamine (Lewis et al., 2006). It is well known that the most extensive dopamine depletion in PD occurs in the caudal part of the putamen and head of the caudate (Smith and Villalba, 2008; Geng et al., 2006; Krabbe et al., 2005; O'Neill et al., 2002; Pitcher et al., 2012). The current shape analysis demonstrated that the striatal regions bearing the greatest magnitude of shape contractions in PD correspond with these areas, suggesting that dopaminergic dysfunction may contribute to the atrophic differences demonstrated herein. Indeed, striatal spine loss has been demonstrated to be highly dependent upon the degree of dopamine depletion in MPTP-treated monkeys, occurring even in the absence of overt motor symptoms (Villalba et al., 2009). Furthermore, striatal spine losses are known to be most severe in the caudal part of the putamen, bearing a remarkable resemblance to the spatial pattern of shape differences described in the current study (Zaja-Milatovic et al., 2005).

In addition to dopamine depletion, a number of other biochemical changes occur within the PD striatum. Several lines of evidence suggest that glutamatergic synaptic and intraspine calcium channel dysregulation may play key roles in PD-related striatal spine pathology (Villalba and Smith, 2010; Burguière et al., 2013; Tian et al., 2010). Previous studies have shown that reducing glutamate release may prevent and/or reverse dopamine depletioninduced dendritic spine loss on medium spiny neurons (Neely et al., 2007; Garcia et al., 2010). In addition, the administration of an L-type calcium channel blocker in an animal model was shown to attenuate these spine losses (Soderstrom et al., 2010; Schuster et al., 2009; Bezard, 2010).

Taken together, these early pathologic features, coupled with PD-related striatal shape differences occurring in recently diagnosed individuals, offer the tantalizing hypothesis that MRI-based striatal shape characteristics may serve as biomarkers for early PD-related changes and for the evaluation of neuroprotective therapies. The sensitivity, specificity, and longitudinal trajectories of shape measurements require further characterization in studies of PD and Parkinsonism subjects.

\subsection{Striatal shape characteristics and clinical associations}

The MoCA is known to be a particularly sensitive instrument for detecting early cognitive decline in PD (Dalrymple-Alford et al., 2010). The finding that striatal volume and shape correlate significantly with MoCA scores in PD subjects is consistent with the notion that the striatum has a complex role in normal cognition as part of several large-scale neural networks. The rostral portions of the putamen and caudate are known to receive projections from the dorsolateral, orbital, and medial prefrontal cortices (Haber, 2003). Similarly, limbic 
projections from the hippocampus and amygdala project to the ventral regions of the striatum. These striatal limbic, associative, and motor circuits are highly integrated and allow for a hierarchical flow of information through the striatal system (Haber et al., 2000). In line with the cognitive correlations demonstrated herein, which are primarily localized to the rostral regions of the putamen and the body and most dorsal extent of the caudate, frontostriatal dysfunction is well documented in PD and may be responsible for some neuropsychological deficits that occur throughout disease progression (Lewis et al., 2003; Brown et al., 1997).

\subsection{Limitations and conclusions}

The cross-sectional nature of the study design and individual variations in the rate of disease progression, coupled with the relatively broad ranges of disease duration and clinical staging, made impossible the assessment of time-related changes. Longitudinal follow-up of this cohort will be necessary to confirm volume and shape alterations as dynamic components of PD progression. In addition, the anatomical precision in segmenting the caudate head was inherently limited by its rostroventral junction with the nucleus accumbens. Although group differences were demonstrated in the most rostral extent of the caudate head, this area may have some degree of overlap with the junction of the nucleus accumbens and thus changes may not be limited exclusively to the caudate head. Lastly, in order for shape analysis to become practical in future clinical trials and medical practice, reliable fully automatic processes are needed. Nevertheless, this study demonstrated regionspecific shape differences measured via MRI within the putamen and caudate of PD patients that are consistent with the known differential pattern of PD-related dopamine depletion in the striatum. We propose that shape characteristics may be useful for reflecting structural changes that occur relatively early in PD.

\section{Supplementary Material}

Refer to Web version on PubMed Central for supplementary material.

\section{Acknowledgments}

This work was supported by NIH NS060722, Penn State Clinical \& Translational Science Institute (NIH UL1 TR000127, TL1 TR000125) and GCRC Construction (C06 RR016499) research grants. We would like to thank the study participants and research coordinators (Mrs. Eleanore Fernandez and Mrs. Brittany Jones) for their contributions to this research. In addition, we would like to acknowledge Mr. Jeffrey Vesek for image data collection, Ms. Zeinab Nasralah for inspection of the anatomical models, and Dr. Richard Mailman for critical review of the manuscript.

\section{References}

UNC NeuroImage Analysis Lab Manual. [Accessed 5-15-2012] IBIS Network. 2007. Online: http:// ibis-network.org/unc/mri/roiprotocols.htm

Apostolova L, Beyer M, Green A, Hwang K, Morra J, Chou YY, Avedissian C, Aarsland D, Janvin C, Larsen J, Cummings J, Thompson P. Hippocampal, caudate, and ventricular changes in Parkinson's disease with and without dementia. Mov. Disord. 2010; 25(6):687-782. [PubMed: 20437538]

Becker JT, Sanders J, Madsen SK, Ragin A, Kingsley L, Maruca V, Cohen B, Goodkin K, Martin E, Miller EN. Subcortical brain atrophy persists even in HAART-regulated HIV disease. Brain Imaging Behav. 2011; 5(2):77-85. [PubMed: 21264551]

Bezard E, et al. Treating Parkinson's disease: preserve the spines!(Commentary on Soderstrom). Eur. J. Neurosci. 2010; 31(3):477. [PubMed: 20105227]

Braak H, Del Tredici K, Rub U, de Vos RA, Jansen Steur EN, Braak E. Staging of brain pathology related to sporadic Parkinson's disease Neurobiol. Aging. 2003; 24(2):197-211. 
Brown GG, Rahill AA, Gorell JM, McDonald C, Brown SJ, Sillanpaa M, Shults C. Validity of the Dementia Rating Scale in assessing cognitive function in Parkinson's disease. J Geriatr Psychiatry Neurol. 1999; 12(4):180-188. [PubMed: 10616865]

Brown LL, Schneider JS, Lidsky TI. Sensory and cognitive functions of the basal ganglia. Curr. Opin. Neurobiol. 1997; 7(2):157-163.

Burguière A, Burke GL, De Bundel D, Valjent E, Roger J, Smolders I, Fagni L, Perroy J. Combination of group I mGlu receptors antagonist with dopaminergic agonists strengthens the synaptic transmission at corticostriatal synapses in culture. Neuropharmacology. 2013; 66(0):151-157. [PubMed: 22465815]

Dalrymple-Alford JC, Macaskill MR, Nakas CT, Livingston L, Graham C, Crucian GP, Melzer TR, Kirwan J, Keenan R, Wells S. The MoCA Well-suited screen for cognitive impairment in Parkinson disease. Neurology. 2010; 75(19):1717-1725. [PubMed: 21060094]

de Lau LML, Breteler M. Epidemiology of Parkinson's disease. Lancet Neurol. 2006; 5(6):525-535. [PubMed: 16713924]

Folstein MF, Folstein SE, McHugh PR. "Mini-mental state" A practical method for grading the cognitive state of patients for the clinician. J Psychiatr. Res. 1975; 12(3):189-198. [PubMed: 1202204]

Garcia BG, Neely MD, Deutch AY. Cortical Regulation of Striatal Medium Spiny Neuron Dendritic Remodeling in Parkinsonism: Modulation of Glutamate Release Reverses Dopamine DepletionInduced Dendritic Spine Loss. Cereb. Cortex. 2010; 20(10):2423-2432. [PubMed: 20118184]

Geng DY, Li YX, Zee CS. Magnetic resonance imaging-based volumetric analysis of basal ganglia nuclei and substantia nigra in patients with Parkinson's disease. Neurosurgery. 2006; 58(2):256262. [PubMed: 16462479]

Haber SN. The primate basal ganglia: parallel and integrative networks. J. Chem. Neuroanat. 2003; 26(4):317-330. [PubMed: 14729134]

Haber SN, Fudge JL, McFarland NR. Striatonigrostriatal pathways in primates form an ascending spiral from the shell to the dorsolateral striatum. J. Neurosci. 2000; 20(6):2369-2382. [PubMed: 10704511]

Hobson P, Meara J. The detection of dementia and cognitive impairment in a community population of elderly people with Parkinson's disease by use of the CAMCOG neuropsychological test. Age Ageing. 1999; 28(1):39-43. [PubMed: 10203203]

Hoops S, Nazem S, Siderowf AD, Duda JE, Xie SX, Stern MB, Weintraub D. Validity of the MoCA and MMSE in the detection of MCI and dementia in Parkinson disease. Neurology. 2009; 73(21): 1738-1745. [PubMed: 19933974]

Hughes AJ, Daniel SE, Kilford L, Lees AJ. Accuracy of clinical diagnosis of idiopathic Parkinson's disease: a clinico-pathological study of 100 cases. J. Neurol. Neurosurg. Psychiatr. 1992; 55(3): 181-184. [PubMed: 1564476]

Ingham CA, Hood SH, van MB, Weenink A, Arbuthnott GW. Morphological changes in the rat neostriatum after unilateral 6-hydroxydopamine injections into the nigrostriatal pathway. Exp. Brain Res. 1993; 93(1):17-27. [PubMed: 7682182]

Jellinger K. Neuropathology of sporadic Parkinson's disease: evaluation and changes of concepts. Mov. Disord. 2012; 27(1):8-38. [PubMed: 22081500]

Jurica PJ, Leitten CL, Mattis S. DRS-2 Dementia Rating Scale-2: Professional manual. Psychological Assessment Resources. 2004

Kish SJ, Shannak K, Hornykiewicz O. Uneven pattern of dopamine loss in the striatum of patients with idiopathic Parkinson's disease Pathophysiologic and clinical implications. N. Engl. J Med. 1988; 318(14):876-880. [PubMed: 3352672]

Krabbe K, Karlsborg M, Hansen A, Werdelin L, Mehlsen J, Larsson HB, Paulson OB. Increased intracranial volume in Parkinson's disease. J. Neurol. Sci. 2005; 239(1):45-52. [PubMed: 16225890]

Lang AE, Obeso JA. Challenges in Parkinson's disease: restoration of the nigrostriatal dopamine system is not enough. Lancet Neurol. 2004; 3(5):309-316. [PubMed: 15099546] 
Langston JW, Widner H, Goetz CG, Brooks D, Fahn S, Freeman T, Watts R. Core assessment program for intracerebral transplantations (CAPIT). Mov Disord. 1992; 7(1):2-13. [PubMed: 1557062]

Lewis MM, Huang X, Nichols DE, Mailman RB. D1 and functionally selective dopamine agonists as neuroprotective agents in Parkinson's disease. CNS. Neurol. Disord. Drug Targets. 2006; 5(3): 345-353. [PubMed: 16787233]

Lewis MM, Smith AB, Styner M, Gu H, Poole R, Zhu H, Li Y, Barbero X, Gouttard S, McKeown MJ, Mailman RB, Huang X. Asymmetrical lateral ventricular enlargement in Parkinson's disease. Eur.J. Neurol. 2009; 16(4):475-481. [PubMed: 19187264]

Lewis SJG, Dove A, Robbins TW, Barker RA, Owen AM. Cognitive impairments in early Parkinson's disease are accompanied by reductions in activity in frontostriatal neural circuitry. J. Neurosci. 2003; 23(15):6351-6356. [PubMed: 12867520]

Maltbie E, Bhatt K, Paniagua B, Smith RG, Graves MM, Mosconi MW, Peterson S, White S, Blocher J, El-Sayed M, Hazlett HC, Styner MA. Asymmetric bias in user guided segmentations of brain structures. Neuroimage. 2012; 59(2):1315-1323. [PubMed: 21889995]

Marras C, Armstrong MJ, Meaney CA, Fox S, Rothberg B, Reginold W, Tang-Wai DF, Gill D, Eslinger PJ, Zadikoff C. Measuring mild cognitive impairment in patients with Parkinson's disease. Mov. Disord. 2013

McNeill TH, Brown SA, Rafols JA, Shoulson I. Atrophy of medium spiny I striatal dendrites in advanced Parkinson's disease. Brain Res. 1988; 455(1):148-152. [PubMed: 3416180]

Monsch AU, Bondi MW, Salmon DP, Butters N, Thal LJ, Hansen LA, Wiederholt WC, Cahn DA, Klauber MR. Clinical validity of the Mattis Dementia Rating Scale in detecting dementia of the Alzheimer type: a double cross-validation and application to a community-dwelling sample. Arch. Neurol. 1995; 52(9):899. [PubMed: 7661728]

Neely MD, Schmidt DE, Deutch AY. Cortical regulation of dopamine depletion-induced dendritic spine loss in striatal medium spiny neurons. Neuroscience. 2007; 149(2):457-464. [PubMed: 17888581]

O’Neill J, Schuff N, Marks WJ Jr, Feiwell R, Aminoff MJ, Weiner MW. Quantitative 1H magnetic resonance spectroscopy and MRI of Parkinson's disease. Mov. Disord. 2002; 17(5):917-927. [PubMed: 12360540]

Otsuka M, Ichiya Y, Kuwabara Y, Hosokawa S, Sasaki M, Yoshida T, Fukumura T, Masuda K, Kato M. Differences in the reduced 18F-Dopa uptakes of the caudate and the putamen in Parkinson's disease: correlations with the three main symptoms. J. Neurol. Sci. 1996; 136(1-2):169-173. [PubMed: 8815166]

Paniagua B, Styner M, Macenko M, Pantazis D, Niethammer M. Local shape analysis using MANCOVA. Insight J. 2009

Pitcher TL, Melzer TR, Macaskill MR, Graham CF, Livingston L, Keenan RJ, Watts R, DalrympleAlford JC, Anderson TJ. Reduced striatal volumes in Parkinson's disease: a magnetic resonance imaging study. Transl.Neurodegener. 2012; 1(1):17. [PubMed: 23210661]

Rodriguez-Oroz M, Jahanshahi M, Krack P, Litvan I, Macias R, Bezard E, Obeso J. Initial clinical manifestations of Parkinson's disease: features and pathophysiological mechanisms. Lancet Neurol. 2009; 8(12):1128-1167. [PubMed: 19909911]

Schuster S, Doudnikoff E, Rylander D, Berthet A, Aubert I, Ittrich C, Bloch B, Cenci MA, Surmeier DJ, Hengerer B. Antagonizing L-type Ca2+ Channel Reduces Development of Abnormal Involuntary Movement in the Rat Model of L-3, 4-Dihydroxyphenylalanine-Induced Dyskinesia. Biol. Psychiatry. 2009; 65(6):518-526. [PubMed: 18947822]

Smith Y, Villalba R. Striatal and extrastriatal dopamine in the basal ganglia: An overview of its anatomical organization in normal and Parkinsonian brains. Mov. Disord. 2008; 23(S3):S534S547. [PubMed: 18781680]

Soderstrom KE, O'Malley JA, Levine ND, Sortwell CE, Collier TJ, Steece-Collier K. Impact of dendritic spine preservation in medium spiny neurons on dopamine graft efficacy and the expression of dyskinesias in parkinsonian rats. Eur. J. Neurosci. 2010; 31(3):478-490. [PubMed: 20105237] 
Stephens B, Mueller AJ, Shering AF, Hood SH, Taggart P, Arbuthnott GW, Bell JE, Kilford L, Kingsbury AE, Daniel SE, Ingham CA. Evidence of a breakdown of corticostriatal connections in Parkinson's disease. Neuroscience. 2005; 132(3):741-754. [PubMed: 15837135]

Styner M, Gerig G, Lieberman J, Jones D, Weinberger D. Statistical shape analysis of neuroanatomical structures based on medial models. Med Image Anal. 2003; 7(3):207-220. [PubMed: 12946464]

Styner M, Lieberman JA, McClure RK, Weinberger DR, Jones DW, Gerig G. Morphometric analysis of lateral ventricles in schizophrenia and healthy controls regarding genetic and disease-specific factors. Proc. Natl. Acad. Sci. U S A. 2005; 102(13):4872-4877. [PubMed: 15772166]

Styner M, Oguz I, Xu S, Brechbuhler C, Pantazis D, Levitt JJ, Shenton ME, Gerig G. Framework for the statistical shape analysis of brain structures using SPHARM-PDM. Insight J. 2006a; (1071): 242. [PubMed: 21941375]

Styner M, Oguz I, Xu S, Brechbuler C, Pantazis D, Gerig G. Statistical shape analysis of brain structures using spharm-pdm. Insight Journal. 2006b

Thompson PM, Hayashi KM, de Zubicaray GI, Janke AL, Rose SE, Semple J, Hong MS, Herman DH, Gravano D, Doddrell DM. Mapping hippocampal and ventricular change in Alzheimer disease. Neuroimage. 2004; 22(4):1754-1766. [PubMed: 15275931]

Tian X, Kai L, Hockberger PE, Wokosin DL, Surmeier DJ. MEF-2 regulates activity-dependent spine loss in striatopallidal medium spiny neurons. Mol. Cell. Neurosci. 2010; 44(1):94-108. [PubMed: 20197093]

Tomlinson CL, Stowe R, Patel S, Rick C, Gray R, Clarke CE. Systematic review of levodopa dose equivalency reporting in Parkinson's disease. Mov Disord. 2010; 25(15):2649-2653. [PubMed: 21069833]

Tustison NJ, Gee JC. Introducing Dice, Jaccard, and other label overlap measures to ITK. Insight Journal. 2009

Vachet C, Perrot E, Ouziel C. AutoSeg 2.1 Documentation. 2001

Van Leemput K, Maes F, Vandermeulen D, Suetens P. Automated model-based tissue classification of MR images of the brain. IEEE Trans. Med. Imaging. 1999; 18(10):897-908. [PubMed: 10628949]

Van LK, Maes F, Vandermeulen D, Suetens P. Automated model-based bias field correction of MR images of the brain. IEEE Trans. Med. Imaging. 1999; 18(10):885-896. [PubMed: 10628948]

Villalba RM, Lee H, Smith Y. Dopaminergic denervation and spine loss in the striatum of MPTPtreated monkeys. Exp. Neurol. 2009; 215(2):220-227. [PubMed: 18977221]

Villalba RM, Smith Y. Striatal spine plasticity in Parkinson's disease. Front Neuroanat. 2010:4. [PubMed: 20428509]

Yekutieli D, Benjamini Y. Resampling-based false discovery rate controlling multiple test procedures for correlated test statistics. J Stat Plan Inference. 1999; 82(1):171-196.

Yushkevich PA, Piven J, Hazlett HC, Smith RG, Ho S, Gee JC, Gerig G. User-guided 3D active contour segmentation of anatomical structures: significantly improved efficiency and reliability. Neuroimage. 2006; 31(3):1116-1128. [PubMed: 16545965]

Zaja-Milatovic S, Milatovic D, Schantz AM, Zhang J, Montine KS, Samii A, Deutch AY, Montine TJ. Dendritic degeneration in neostriatal medium spiny neurons in Parkinson disease. Neurology. 2005; 64(3):545-457. [PubMed: 15699393] 


\section{Vector field}

\section{P-value}
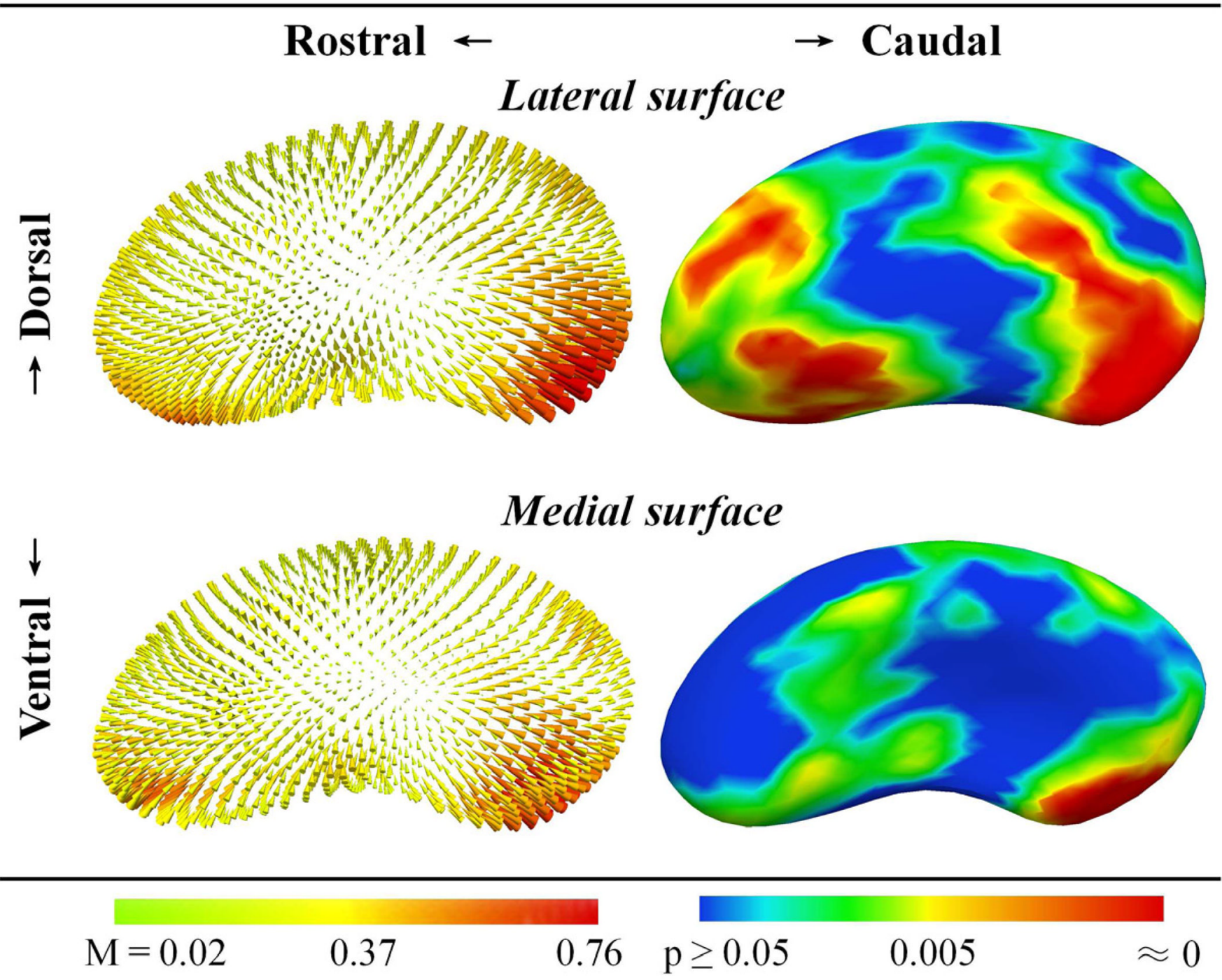

Figure 1.

Putamen shape differences between 40 PD and 40 control subjects. Displacement vectors indicate the direction and magnitude $(\mathrm{M})$ of distance from the average control surface to the average PD surface. FDR-adjusted p-values are for comparisons between PD and control structures, adjusted for age and gender. The greatest magnitudes of surface contraction were located on the caudal putamen. Structures were pooled bilaterally and scaled to account for variations in intracranial volume. 


\section{Vector field $\quad$ P-value}
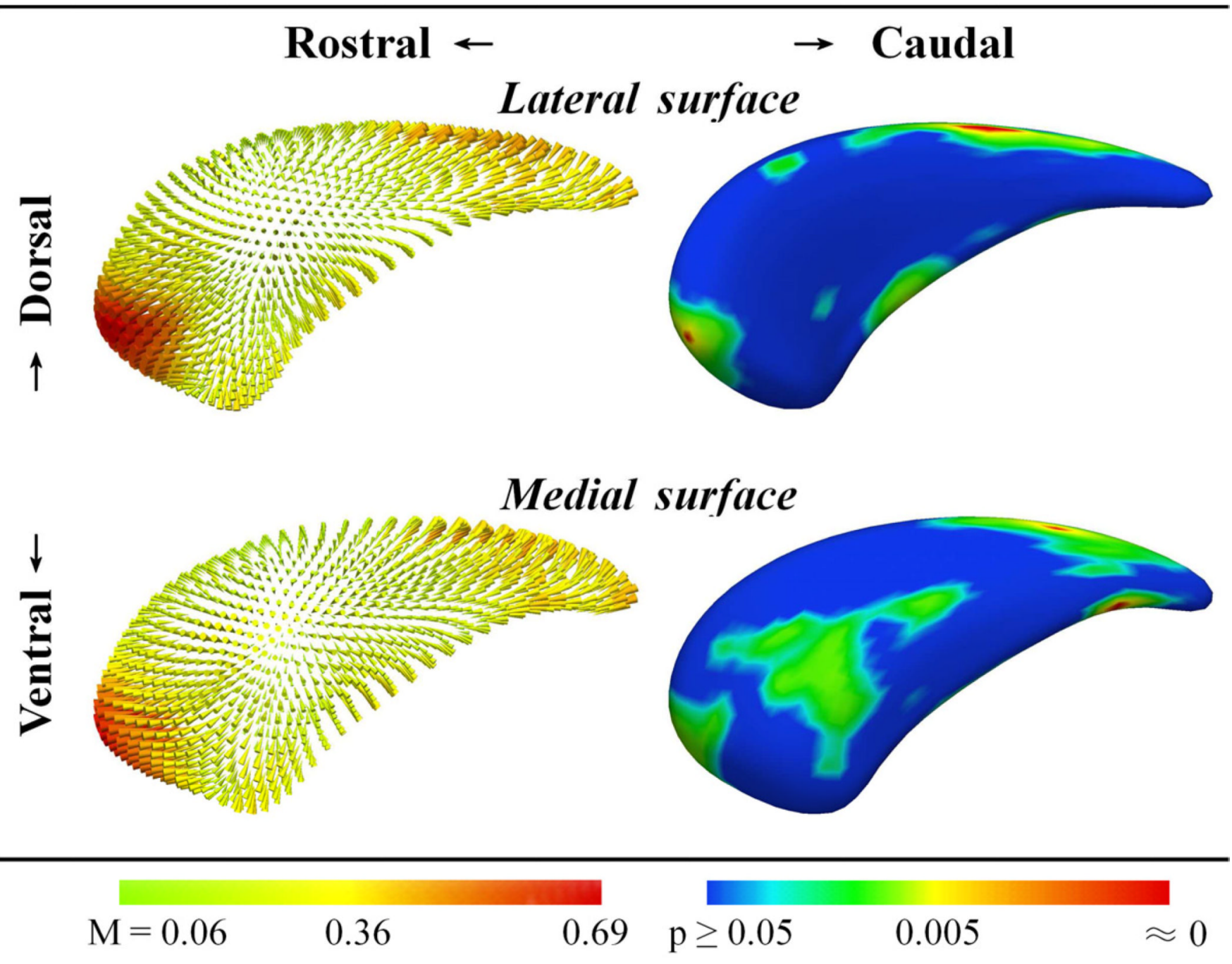

Figure 2.

Caudate shape differences between 40 PD and 40 control subjects. Displacement vectors indicate the direction and magnitude $(\mathrm{M})$ of distance from the average control surface to the average PD surface. FDR-adjusted p-values are for comparisons between PD and control structures, adjusted for age and gender. The greatest magnitudes of surface contraction were located on the rostral caudate head. Structures were pooled bilaterally and scaled to account for variations in intracranial volume. 


\section{Pearson's R P-value}
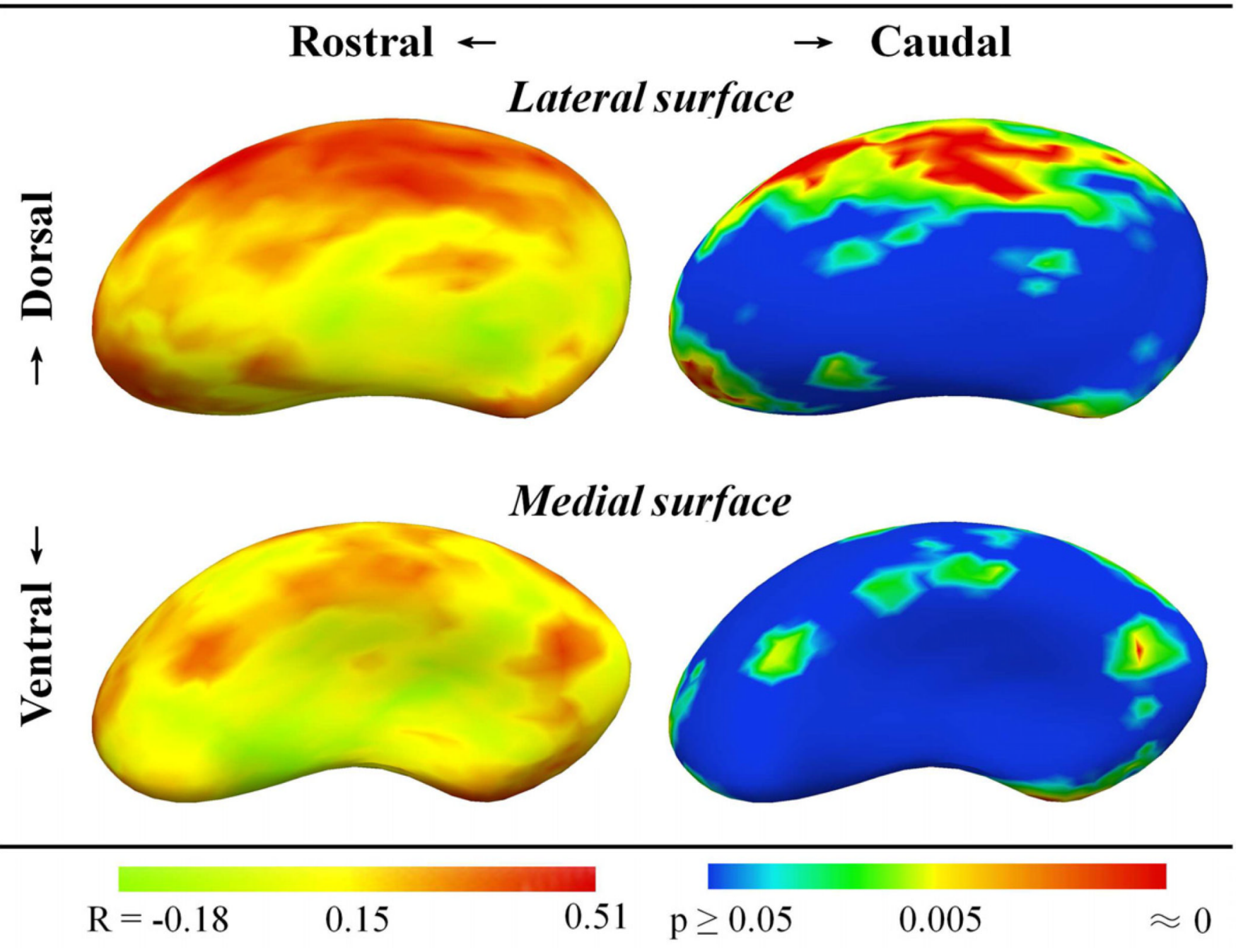

Figure 3.

Correlation of putamen shape and MoCA scores in 40 PD subjects. Worsening MoCA scores are related to contraction of the dorsal and rostroventral surfaces. Pearson's $\mathrm{R}$ and FDR-adjusted p-value maps represent correlations between signed distance from average surface at each surface point and MoCA score residuals after regression for age and gender. Structures were pooled bilaterally and scaled to account for variations in intracranial volume. 


\section{Pearson's R P-value}
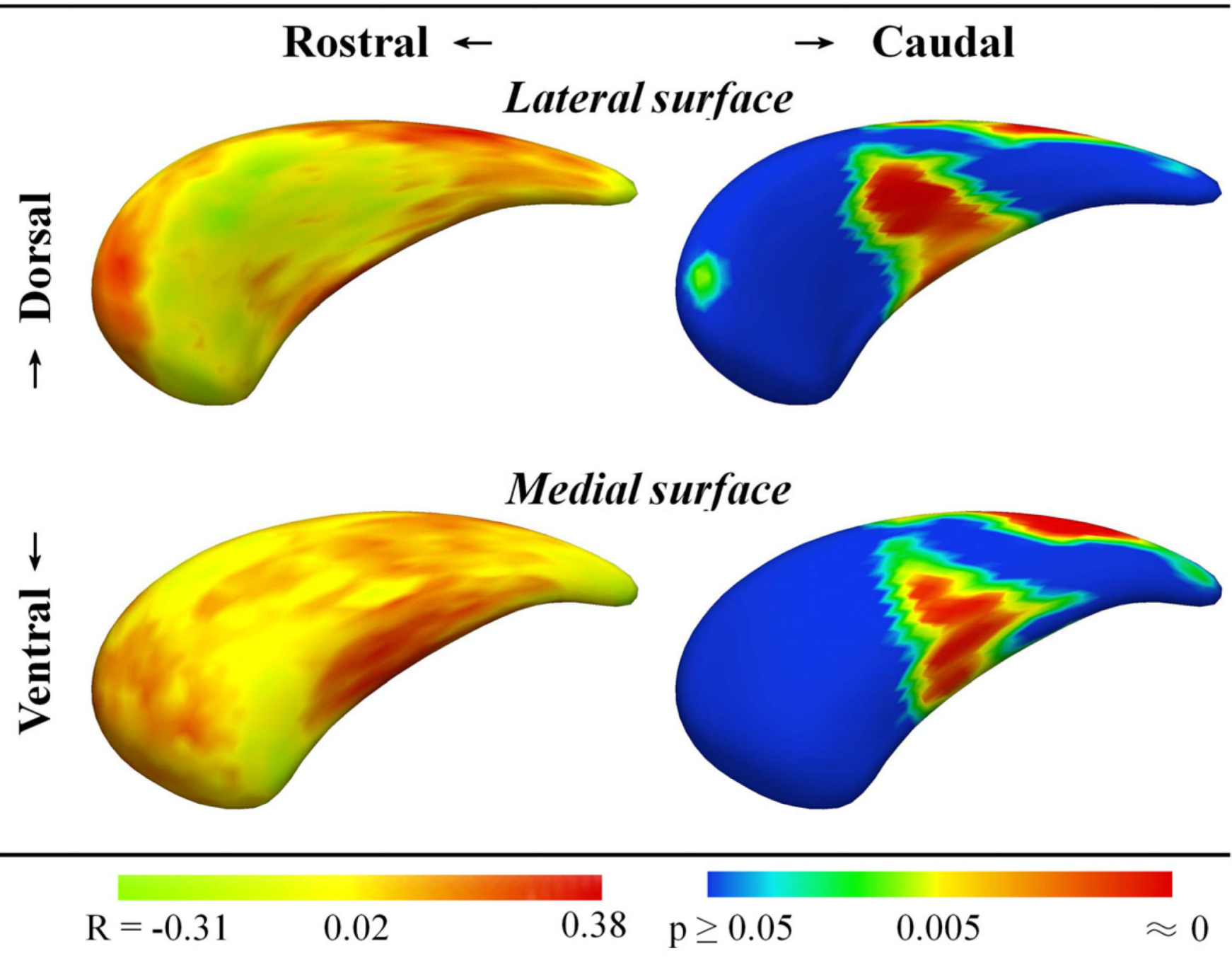

Figure 4.

Correlation of caudate shape and MoCA scores in 40 PD subjects. Worsening MoCA scores are related to contractions on the dorsal surface and body of the caudate. Pearson's R and FDR-adjusted $\mathrm{p}$-value maps represent correlations between signed distance from average surface at each surface point and MoCA score residuals after regression for age and gender. Structures were pooled bilaterally and scaled to account for variations in intracranial volume. 
Table 1

Demographic and clinical information of PD and control subjects

\begin{tabular}{lcrc}
\hline & Control (SD) & PD (SD) & P-Value \\
\hline Total N [F/M] & $40[20 / 20]$ & $40[20 / 20]$ & NA \\
Age & $59.4(7.8)$ & $61.3(7.8)$ & $0.2670^{a}$ \\
Years Since Diagnosis & NA & $4.1(4.2)$ & NA \\
Motor Measurements & & & \\
Hoehn-Yahr Stage & $0(0)$ & $1.8(0.6)$ & $<0.0001^{b}$ \\
UPDRS-III & $1.1(1.26)$ & $22.6(14.7)$ & $<0.0001^{b}$ \\
LEDD (mg) & $\mathrm{NA}$ & $465.3(376.8)$ & $\mathrm{NA}$ \\
Cognitive Measurements & & & \\
DRS2 & $141.2(2.3)$ & $140.2(3.6)$ & $0.1447^{a}$ \\
MMSE & $29.5(0.8)$ & $29.2(1.2)$ & $0.1840^{b}$ \\
MoCA & $25.8(2.4)$ & $25.6(2.9)$ & $0.7360^{b}$ \\
\hline
\end{tabular}

All values are presented as group means and standard deviations (SD), except for the number and gender of subjects. Motor and cognitive measurements in PD subjects were obtained in the off-medication state (see Methods).

${ }^{a} \mathrm{P}$-values calculated using two-sample t-tests with pooled variance.

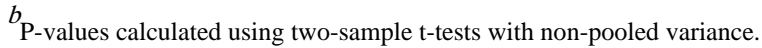

Abbreviations: DRS2 = Dementia Rating Scale-2; LEDD = levodopa equivalent daily dose; MMSE = Mini Mental State Examination; MoCA = Montreal Cognitive Assessment; UPDRS-III = Unified Parkinson's Disease Rating Scale section III. 
Table 2

Striatal volumes (percent of intracranial volume) in PD and control subjects

\begin{tabular}{lllll}
\hline \multicolumn{7}{c}{ PD } & Control & PD/Control (\%) & P-Value \\
\hline \multicolumn{1}{c}{ Analysis of all subjects $\boldsymbol{a}$} & & & \\
$\mathrm{N}$ & 40 & 40 & & \\
Putamen & $0.306(0.00434)$ & $0.330(0.00434)$ & 92.9 & 0.0003 \\
Caudate & $0.228(0.00316)$ & $0.245(0.00316)$ & 93.0 & 0.0003 \\
\hline Subgroup analysis $\boldsymbol{b}$ & & & \\
PD subjects & $0.1-2.3$ years since diagnosis & & \\
$\mathrm{N}$ & 20 & 40 & & \\
Putamen & $0.306(0.00653)$ & $0.331(0.00493)$ & 92.5 & 0.0024 \\
Caudate & $0.232(0.00551)$ & $0.247(0.00420)$ & 94.0 & 0.0036 \\
PD subjects & $0.1-1.1$ years since diagnosis & & \\
$\mathrm{N}$ & 10 & 40 & & \\
Putamen & $0.302(0.00974)$ & $0.331(0.00493)$ & 91.3 & 0.0061 \\
Caudate & $0.234(0.00698)$ & $0.247(0.00420)$ & 94.7 & 0.0248 \\
\hline
\end{tabular}

${ }^{a}$ Adjusted mean scaled volumes and standard errors.

${ }^{b}$ Unadjusted mean scaled volumes and standard errors.

All p-values are adjusted for age and gender. 
Table 3

Partial correlation coefficients and p-values between scaled striatal volumes and clinical measurements in PD subjects

\begin{tabular}{|l|c|c|}
\hline & Putamen & Caudate \\
\hline Hoehn-Yahr Stage & $\begin{array}{c}-0.13^{a} \\
(0.4785)\end{array}$ & $\begin{array}{c}-0.10^{a} \\
(0.5837)\end{array}$ \\
\hline LEDD & $\begin{array}{c}0.14^{b} \\
(0.4141)\end{array}$ & $\begin{array}{c}-0.25^{b} \\
(0.1289)\end{array}$ \\
\hline UPDRS-III & $\begin{array}{c}0.11^{b} \\
(0.5054)\end{array}$ & $\begin{array}{c}-0.03^{b} \\
(0.8666)\end{array}$ \\
\hline DRS2 & $\begin{array}{c}0.04^{b} \\
(0.8230)\end{array}$ & $\begin{array}{c}0.17^{b} \\
(0.3059)\end{array}$ \\
\hline MMSE & $\begin{array}{c}0.11^{b} \\
(0.5240)\end{array}$ & $\begin{array}{c}0.27^{b} \\
(0.1093)\end{array}$ \\
\hline MoCA & $\begin{array}{c}0.53^{b} \\
(0.0006)\end{array}$ & $\begin{array}{c}0.32^{b} \\
(0.0503)\end{array}$ \\
\hline
\end{tabular}

${ }^{a}$ Spearman's partial correlation coefficients after adjustment for age and gender.

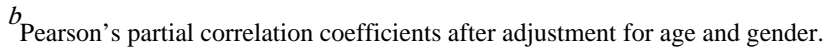

Significant after Bonferroni correction for multiple comparisons $(\mathrm{p}=0.0072)$.

Abbreviations: DRS2 $=$ Dementia Rating Scale-2 $;$ LEDD = levodopa equivalent daily dose $;$ MMSE $=$ Mini Mental State Examination; MoCA $=$ Montreal Cognitive Assessment; UPDRS-III = Unified Parkinson's Disease Rating Scale section III. 